\title{
Integrating emerging technologies into chemical safety assessment: progress since the 2012 report of the expert panel on the integrated testing of pesticides
}

\author{
Leonard Ritter* \\ School of Environmental Sciences, \\ University of Guelph, \\ Room 2114, Bovey Building, \\ Guelph, ON N1G 2W1, Canada \\ Email: 1ritter@uoguelph.ca \\ *Corresponding author
}

\section{Sam Kacew}

McLaughlin Centre for Population Health Risk Assessment, University of Ottawa,

Ontario K1G 3Z7, Canada

Email: sam.kacew@uottawa.ca

\section{Daniel Krewski}

McLaughlin Centre for Population Health Risk Assessment, University of Ottawa,

Ontario K1G 3Z7, Canada

and

Risk Science International,

Ottawa, Ontario, Canada

Email: dkrewski@uottawa.ca

\begin{abstract}
Governments need to categorise tens of thousands of data-poor chemicals in order to better inform human health risk assessment. Pesticide active ingredients have contributed considerably to our understanding of the toxicological mechanisms; however, in order to move forward there is a pressing need for testing that is faster and less expensive based upon the chemical specific mode-of-action (MOA). Currently, a comprehensive set of these alternative methods does not yet exist, although the state of the science is rapidly evolving. The next two to ten years will see a global progression towards the use of integrated testing strategies (ITS) in decision-making for both data-rich and data-poor chemicals. Regulatory deployment of integrated approaches to testing and assessment (IATA) will depend upon the types of chemicals and the nature of the decision-making process by regulatory authorities. Regulators need to recognise that adoption of IATA strategies would require the engagement and approval of public stakeholders in order to alleviate concerns regarding potential adverse risks to human health and the environment. The new approach cannot be used to simply streamline processes or sacrifice human and environmental safety for social or economic benefits.
\end{abstract}


Keywords: pesticides, toxicity testing; integrated approaches to testing and assessment; IATA; integrated testing strategies; ITS; chemical safety assessments.

Reference to this paper should be made as follows: Ritter, L., Kacew, S. and Krewski, D. (2017) 'Integrating emerging technologies into chemical safety assessment: progress since the 2012 report of the expert panel on the integrated testing of pesticides', Int. J. Risk Assessment and Management, Vol. 20, Nos. 1/2/3, pp.46-58.

Biographical notes: Leonard Ritter is a Professor Emeritus of Toxicology in the School of Environmental Sciences at the University of Guelph. He has a long-standing interest in the toxicology and public health impact of a broad range of food and environmental contaminants, including pesticide residues. He has served as the Chief of the Pesticides Division at Health Canada, as a member of the WHO/FAO Joint Expert Committee on Food Additives and also chaired the Council of Canadian Academies expert panel on the future of toxicity testing of pesticides. He is a Fellow of the Academy of Toxicological Sciences.

Sam Kacew is an Associate Director of Toxicology, McLaughlin Centre for Population Health Risk Assessment at the University of Ottawa. He is currently the Editor-in-Chief of the Journal of Toxicology and Environmental Health, Part A, Current Issue, and Journal of Toxicology and Environmental Health, Part B Critical Reviews, North American Editor, Toxicological and Environmental Chemistry and Associate Editor of Toxicology and Applied Pharmacology. He is a member of the Board on Environmental Studies and Toxicology (BEST) of the National Academy of Sciences, USA. He is a member of the Science Advisory Council (SAC) of the National American Flame Retardant Association (NAFRA). He was a member of the Board of Review for Siloxane D5 appointed by the Minister of Environment of Canada. His research interests include general toxicology including renal, hepatic and pulmonary toxicology. He has edited several texts on paediatric toxicology.

Daniel Krewski is a Professor in the School of Epidemiology, Public Health and Preventive Medicine at the University of Ottawa, where he also serves as the Scientific Director of the McLaughlin Centre for Population Health Risk Assessment. His research interests include epidemiology, biostatistics, risk assessment, and risk management. He is a Fellow of the Society for Risk Analysis, the American Statistical Association, and a National Affiliate of the US National Academy of Sciences. He holds the Natural Sciences and Engineering Research Council of Canada Chair in Risk Science at the University of Ottawa.

This paper is a revised and expanded version of a paper entitled 'Integrated testing of pesticides: a Canadian perspective' presented at Risk Science in the 21st Century, Ottawa, Ontario, Canada, 4-6 March 2013.

\section{Introduction}

Pesticide safety is a health and environmental concern throughout the world. Pesticides are widely used in Canada in agriculture, industrial and urban applications to enhance food production to meet the demands of society. The safety of pesticides has attracted 
public scrutiny and attention, particularly in urban and residential settings where many Canadian provinces have already implemented bans or restrictions on their use. Pesticides contain active ingredients that function to mitigate the target pest. Pesticide formulations include components that dissolve, add stability and enhance the effectiveness of the active ingredient. The active ingredients are rigorously regulated compounds, the safety of which is supported by a comprehensive toxicological assessment (largely laboratory studies) that follows a regimen similar to the preclinical safety assessment of a prescription drug. However, extensive evaluation of the active ingredients in pesticides does not extend to the other components of the pesticide product (most notably the formulants).

Extensive toxicity testing data on pesticide 'active ingredients' are available. Nonetheless, current testing protocols are both costly and time-consuming resulting in a significant backlog of non-pesticide active ingredient chemicals that require testing.

Scientific research has evolved significantly so that our understanding of molecular mechanism has increased. These advancements have not been widely applied to toxicity testing protocols required for regulatory decision-making (Seidle and Stephens, 2009). Many of these standardised tests have remained the same for decades. Traditionally, past toxicological protocols were not intended to produce data relating to molecular mechanisms and perturbation pathways. As a result, there is a need for new scientific techniques that provide mechanism-based, chemical-specific toxicological information. This new paradigm shift in toxicology will transform risk assessment into a science that incorporates knowledge of the toxicity pathways and mechanisms by which chemicals exert their adverse health effects.

In 2012, Canadian Council of Academies published a report based upon the opinions of the expert panel on the integrated testing of pesticides. This paper provides a summary of the report along with scientific advances since the report had been written.

\section{Charge to the panel}

The Expert Panel on the Integrated Testing of Pesticides was established by the Council of Canadian Academies at the request of the Pest Management Regulatory Agency (PMRA).

The panel report (CCA, 2012) is the opinion of the expert panel and not the official position of the PMRA. The panel was asked to assess and report on the scientific status of the use of integrated testing strategies (ITS) for the regulatory risk assessment of pesticides. Specifically, the panel was asked by the Government of Canada to address three specific questions (CCA, 2012):

- What is the state of the science of the tools and data sources associated with ITS?

- What is the current status of the use of ITS for the risk assessment of pesticides, pharmaceuticals, industrial chemicals, and other chemical substances by regulatory agencies around the world?

- Could there be potential impacts on the public's perception and confidence in regulatory risk assessment and risk management decisions for pesticides if ITS were implemented? 
In order to address these questions, the panel structured its deliberations and report around three pillars:

1 where possible, distinguish between data-rich and data-poor chemicals

2 populate with examples to provide real-life context

3 discuss near-, medium-, and long-term options.

The gap between toxicological testing capacity and the insatiable growing global appetite for better and faster means to assess the safety of chemicals needs to be recognised. The panel focused its assessment on new approaches which would be more predictive, more reliable, faster, and less expensive. In addition, the new approaches need to provide mechanism-based information regarding the underlying toxicity of a chemical in order to inform risk assessment, and identify the cellular response pathway that, when sufficiently perturbed, is expected to result in an adverse health effect.

\section{State of the science}

Integrated approaches to testing and assessment (IATA) aim to change an outdated approach to toxicology assessment. Toxicology testing will focus on mechanism of action explaining 'how' it happens rather than describing 'what' happens. It will be clear that the focus needs to shift away from endpoint toxicology and move toward an understanding of the mechanisms which lead to adverse outcomes (CCA, 2012). Several different approaches to IATA have been discussed in a review publication by Rovida et al. (2015). These include

a weight of evidence (WoE) which examines the evidence presented by a number of independent assays to make a decision

b battery of tests which relates to a series of tests that all need to be performed to replace in vivo testing

c tiered strategy testing where the first tier of testing decides what the next tier of testing would involve.

IATA could involve a combination of some or all of these approaches in addition to non-test methods such as read-across and in silico modelling. The focus is on mechanism and the existence of an adverse outcome pathway (AOP), where key events to an in vivo endpoint of regulatory interest are fundamental to the use of any IATA. An example of a well understood AOP is the pathway that triggers skin sensitisaton. This mode-of-action (MOA) and the key events in the pathway have been well mapped out (Rovida et al., 2015). Advances in other scientific disciplines are also furthering development of new scientific tools, which can be applied to toxicological evaluation. At the core of this scientific evolution are the fields of systems biology and computational toxicology. IATA uses scientific tools and techniques from a wide variety of disciplines. Krewski et al. (2014) tabulated a number of tools and techniques that have advanced over the years and are currently in use for several case studies. FutureTox II, a workshop held by the Society of Toxicology (SOT) in 2014, summarised the current status of in vitro and in silico predictive methods (Elmore et al., 2014). These scientific tools are at different stages of 
completeness and are constantly evolving and improving. Some of these new approaches utilise computational approaches such as physiologically based pharmacokinetic (PBPK) modelling to weigh existing toxicity data, while others generate new toxicity data employing a vast array of in vitro approaches such as high-throughput screening (HTS) data. The US EPA have spent millions generating new in vitro HTS data in the ToxCast I, II and III programs (Knudsen et al., 2011; Sipes et al., 2013; Judson et al., 2014) as well developing new tools to integrate all types of data in a meaningful manner (Reif et al., 2010, 2013). ToxPi GUI, a computational tool launched by the US EPA is an example of an interface that integrates all types of data from multiple sources into a visual format for chemical prioritisation (Reif et al., 2013).

The applicability and ultimate acceptability of these tools in regulatory environments will be directly related to the engagement and participation of the international regulatory community in their development. The implementation and performance of proof-of-concept studies will bring confidence and familiarity to the new methods of generating data. A workshop held in Italy in 2013 brought together a group of experts from industry, the scientific community as well as regulatory, validation and government agency to discuss the current available technology for IATA, future directions and limitations. Skin sensitisation was used as a case study to demonstrate how IATA can be used, optimised and validated in a regulatory context (Rovida et al., 2015). An ideal IATA would be adaptable incorporating new types of data, as it becomes available and when required.

Proof-of-concept studies highlight the significance of comprehensive and computable data. Andersen et al. (2011) outlined the advantages of a case study approach. Subsequently, important advances in these proof-of-concept studies have evolved to the point that IATA tools can now be used to make predictions about several acute toxicity endpoints. A recent workshop (Somma Lombardo, Italy, March 2-7th, 2014) entitled 'Advancing AOPs for Integrated Toxicology and Regulatory Applications' discussed a case study example of the AOP of organophosphate and carbamate insecticides, where acetylcholinesterase (AChE) inhibition eventually led to acute mortality (Tollefsen et al., 2014). This AOP has been clearly established in several species along with initiating and key events for adverse outcomes (Russom et al., 2014) and illustrates that a recognised AOP could be used for some regulatory purposes without testing of every key event. Other examples of proof-of-concept studies investigated whether the well-defined AOPs relevant in animals/non-animal systems translate into equivalent hazards in humans. Case studies of AOPs for liver cancer to identify human relevant MOA and key events for:

1 aryl hydrocarbon receptor (AHR) (Budinsky et al., 2014)

2 peroxisome proliferator-activated receptor alpha (PPAR $\alpha)$ (Corton et al., 2014)

3 constitutive androstane receptor (CAR) and pregnane $\mathrm{X}$ receptor (PXR) (Elcombe et al., 2014) were recently published.

Scientists did not consider the latter two AOPs to be MOAs in humans for liver carcinogenicity.

In the short-term, IATA approaches to evaluate acute toxicity pathway perturbations will likely become available. But progress will be slower in developing non-animal (non-in vivo) replacement approaches to long-term endpoints, such as carcinogenicity and reproductive toxicity. In the European research programme, the SEURAT-1 project has embraced a MOA framework to improve non-animal toxicity assessment and adopted a 
case study approach that has shaped their research. A 'conceptual framework' has emerged, built on designing an integrated assessment strategy for case studies (Datson et al., 2015; Gocht et al., 2015). In the long-term, predictions based on the complete understanding of toxicological mechanism would be the ultimate goal, but in the near term these predictions need to be verified with data-rich case studies (Gocht et al., 2015). Realistically, it is likely that it will be at least a decade, and perhaps longer, before IATA approaches will have achieved the requisite technical refinement and won the confidence and endorsement of the regulatory communities.

IATA tools can already be applied in a regulatory context for data-poor chemicals for which classical in vivo test methods cannot be implemented on any practical level. For example, Thomas et al. (2013) developed a margin-of-exposure (MOE) framework where data-poor chemicals are categorised based upon comparing extrapolated in vitro assay data (IVIVE and BPAD) to estimated exposures levels. An overlap of the two calculated doses determines whether further testing is required. With sufficient pharmacokinetic (PK) data, dosage for data-poor chemicals can be predicted from HTS assays (Wambaugh et al., 2013; Wetmore et al., 2013, 2015). In this respect, IATA approaches would seem to represent a substantial improvement of the safety database for data-poor chemicals for which regulatory decisions are often made on the basis of little or no primary data.

The panel recognised that rapid advances in in silico and in vitro methodology now implies that thousands of chemical entities can at least be screened on a daily basis, compared to only 10-100 chemical entities that can be evaluated on an annual basis utilising conventional in vivo methods (Figure 1).

Figure 1 Increasing throughput capacity

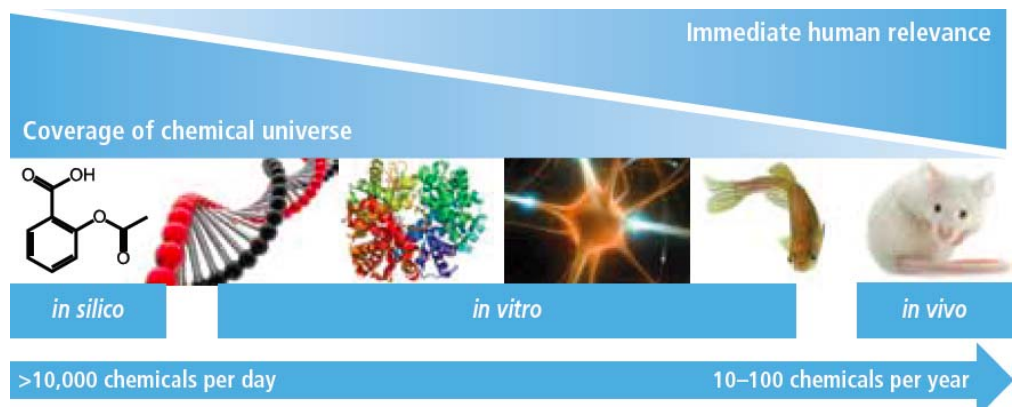

Source: CCA (2012) (C) 2012 Council of Canadian Academies with permission

The panel also recognised that IATA would:

1 provide a pragmatic approach to address existing issues

2 require a fundamental paradigm shift and represent a bridging paradigm

3 transition away from one-size-fits-all whereby all chemicals, regardless of relevance, are subjected to the same suite of studies, to a refined and focused testing approach specific to the chemical entity 
4 focus testing on endpoints of concern rather than assessing the same endpoints for all chemicals

5 leverage state-of-the-art tools and techniques, and includes advances in systems biology and computational toxicology.

As illustrated in Figure 2, the panel was of the view that in silico and in vitro methods could be used to initially screen large numbers of chemicals while more toxicologically advanced in vitro and in vivo approaches could then be employed to identify hazards for high priority chemical entities identified in the screening process.

Figure 2 The hazard identification paradigm

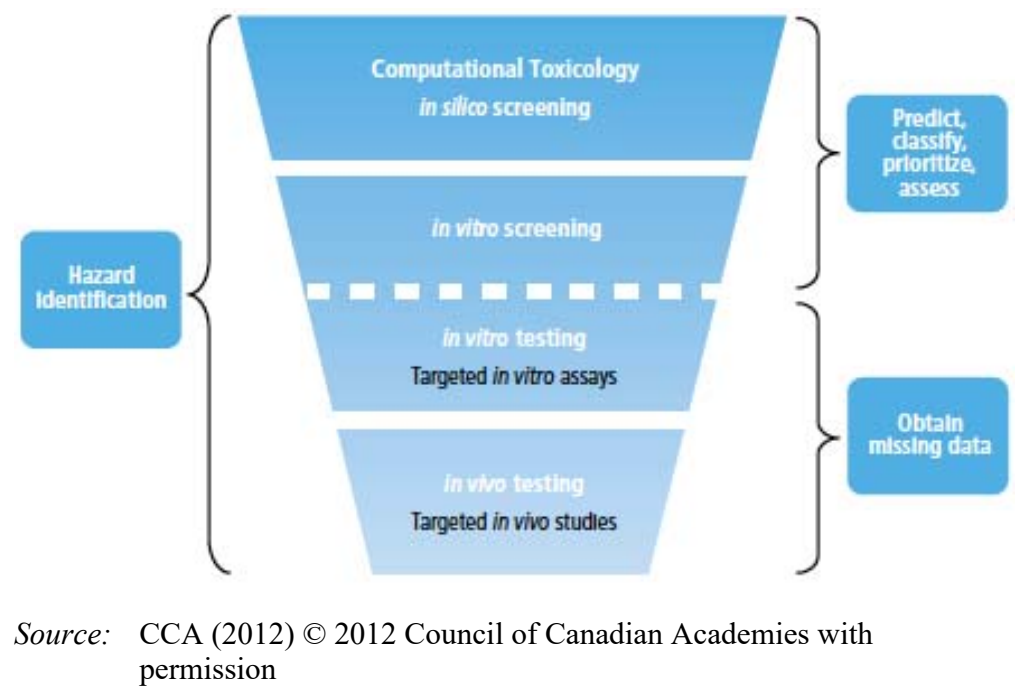

This approach has been adopted to varying degrees by several European, Canadian and US regulatory agencies (Interagency Coordinating Committee on the Validation of Alternative Methods - ICCVAM, 2006a, 2006b).

\subsection{What is the current status of the use of ITS for the risk assessment of pesticides, pharmaceuticals, industrial chemicals, and other chemical substances by regulatory agencies around the world?}

While there are numerous examples where components of IATA have been applied in the safety assessment of industrial chemicals and personal care products, there does not appear to be any apparent instance where there has been a disciplined hierarchal application of IATA in a regulatory context. The Organisation for Economic Co-operation and Development (OECD), with its member countries, are making an effort to establish universal guidelines for validation of tests that represent IATA-based formats. The OECD has recently published guidance documents on IATA for skin sensitisation, corrosion and irritation (OECD, 2012a, 2012b, 2014). It appears clear that the regulatory application of the principles of IATA will vary as a function of the type of chemical in question and the nature of the decision-making processes that the data are intended to inform. For those chemicals which are characterised by a paucity of safety data - the so-called data-poor chemicals - the lack of traditional toxicological data to 
assess safety may be the critical impetus for a new approach such as the application of IATA. A consensus among the scientific, industrial and regulatory communities would move IATA forward so that this type of testing could be used in a regulatory context (Rovina et al., 2015).

In contrast, data-rich chemicals such as pesticides and prescription drugs, are already the subjects of an extensive array of elaborate and complex toxicity studies. Thus establishing the relevance and applicability of the 'new' science will invariably first be met with cynicism and the adoption of the principles of IATA will take longer and will almost certainly be influenced by our ability to build and establish trust in the new and novel methods which lack the history and track record of more conventional laboratory protocols which have now been in place, largely unchanged, for almost four decades. While the adoption of IATA strategies might contribute significantly to our understanding of the toxic potential of chemicals and enhance the reliability of the test outcome, there can be little doubt that widespread application of IATA will be met with doubts and is not likely to occur in the short term.

The basic underpinning of the IATA approach is its reliance on the use of all existing data in order to identify and address data gaps, thereby informing evidence-based decision-making. It is therefore axiomatic that if the concept of an IATA is rooted in an understanding of the fundamental biological mechanisms that are the basis of a toxicological outcome, adoption of IATA strategies could lead to a more efficient testing template so that only those chemicals and endpoints for which there is a biological imperative for a particular chemical entity would be subjected to a costly and time consuming in vivo test.

IATA necessitates a new approach to the development of test protocols and acceptance of these new methods by the regulatory community. It is critically important to recognise that alternative methods target specific cellular or physiological pathways. As such, new methods such as a HTS assay cannot be validated or directly compared with in vivo data in a one-for-one approach. Simply stated, IATA approaches target fundamentally different endpoints than classical in vivo studies, thereby rendering a one for one comparison between results from a classical toxicological protocol to an IATA derived endpoint entirely and scientifically implausible. The AOP approach sanctions the use of a suite of models or assays (and subsequent databases) that are designed to target key events along toxicity pathways. The next tier of toxicity testing would be based on the previous assay/dataset. Comparison of test outcomes for the different tiers with perturbations of toxicity pathways need to be based on known MOA.

Thus far, computational toxicology, has been utilised predominantly in the regulatory decision-making for data-poor chemicals, pesticide formulants - the non-active components of pesticide formulations which typically include solvents, emulsifiers and other components which, by themselves, exert no apparent pesticidal activity - are typical of these types of chemical entities. As such, IATA continue to develop and gain acceptance within the scientific and regulatory communities, and it can reasonably be expected to see increased use of ITS in decision making, for a broad range of chemicals, including both data-poor and data-rich chemical entities. Integration of these novel and evolving scientific tools and technologies with existing data is a practical approach which could be used to strengthen the current regulatory model and bridge the conversion from in vivo research focused on apical studies to a hypothesis-driven approach, which focuses on the pathway or underlying mechanism of the adverse effect. 
Although components of IATA have been used in a regulatory context, and the adoption of IATA strategies might refine and streamline testing of chemicals as well as enhance the reliability of the outcome, there are no known applications that can completely replace the entire current test requirements. As a result, it is unlikely that we can expect adoption of IATA in the short-term. Importantly, adoption and implementation of IATA will require a fundamental shift in the toxicological paradigm away from the evaluation of apical endpoints and toward an understanding of the AOP. Similarly, capacity building will also be crucial to develop proficiency, trust, and familiarity with the new methods and data.

\subsection{Could there be potential impacts on the public's perception and confidence in regulatory risk assessment and risk management decisions for pesticides if ITS were implemented?}

Changes in regulations always raise concerns for both the regulated industry and for the general public who are the subjects to be protected by the evolving regulations. There can be little doubt that major concerns will be a characteristic of the implementation of the changes, which the new IATA tools will bring. Concerns will focus on whether these changes enhance the ability to identify the most important adverse risks to human health and environment or whether they compromise this ability in the interest of streamlining the government regulatory machinery and enhancing business profitability. It can reasonably be anticipated that the public will demand assurances that the new methods reduce overall uncertainties in the assessment of chemical risk and, where new uncertainties are introduced, that these will be addressed in ways, which are at least as precautionary as afforded by the current toxicological approach.

Given the broadly based concern with pesticide safety, it is reasonable to expect that any change in regulatory requirements would generate public concerns about whether these new processes are sufficient to protect human health. Public reassurance should be a major focus for regulators. Any changes made to the existing paradigm of safety assessment need to provide proof that the new processes in place involve reliable assessments of health and environmental risks rather than sacrifice safety for social or economic benefits. In addition, streamlining the processes should be regarded as a means of demonstrating that the back log of thousands of data-poor chemicals includes a means of prioritisation whereas none was in place before.

The pros and cons of the current and new system of chemical risk assessment needs to be transparent and understandable to general public. Many concerned stakeholders will evaluate any change against historical benchmark doses irrespective of the imperfections and uncertainties already inherent in the current testing paradigm.

Regulators will need to consider issues of key importance to the general public. For example, will the new IATA tools be used to supplement (and thus strengthen) the current system or to replace it?; What scientific uncertainties in the current system of chemicals management are reduced by the implementation of new IATA tools?; What new uncertainties are introduced by the use of these tools?; How will the changes in the scientific uncertainties be handled in the regulatory process?; Will the current 'margins of safety' used in the in vivo toxicity testing regime be reduced?; And will safety standard thresholds be reduced with respect to certain kinds of chemicals? 
The CCA Panel report indicates that the new IATA tools should only be introduced in a supplementary manner into the regulatory system. This would test the reliability of the new methods, and increase public confidence in the regulation of chemical pesticides.

Transparency would be a decisive strategy in building public confidence in the regulatory system as IATA tools are employed. It is important that the new tools are accompanied by proof-of-concept case studies which provide clear and accurate demonstration that IATA works.

The panel was of the view that:

1 transparency is critical

2 the public will need to be reassured that the new methods are at least as

precautionary as those in the current system

3 in order to build public confidence, it is important that IATA be introduced incrementally and the new tools explained as clearly and accurately as possible.

\section{Conclusions of the panel}

The panel concluded that the transition to an integrated approach to toxicity testing could significantly enhance the existing regulatory framework, particularly for data-poor but also for data-rich chemicals and this paradigm shift will necessitate a new and transparent approach to test development, validation, and regulatory acceptance on a national and international scale.

The active participation and meaningful engagement on the part of regulatory authorities, the regulated community, and other stakeholders in order to shape and adopt new approaches would be needed.

In addition, the panel also concluded that IATA would move testing away from describing the end point of what happens, towards an explanation of the mechanism of how it happens. The problem of the backlog of data-poor chemicals would also be addressed. IATA would permit the integration of new science into the existing regulatory framework and augment the reliability of existing testing practices.

IATA strength lies in the breadth of information that is used to understand a toxicological profile of a chemical.

Importantly, the panel also noted that it was not aware of a complete set of alternative methods that could replace the entire testing paradigm today (even for data-poor chemicals), but noted that the state of the science is evolving rapidly. With the continued development of such tools and approaches, the panel expects to see increased use of ITS in decision making, with an eventual adaptation to inform decisions involving data-rich chemicals.

While the panel felt that adoption of IATA strategies will be slow, several initiatives are ready, or nearly ready in the short term, for implementation. The status of various initiatives is illustrated in Figure 3. 
Figure 3 The road ahead



Source: CCA (2012); (C) 2012 Council of Canadian Academies with permission

\section{References}

Andersen, M.E., Clewell III, H.J., Carmichael, P.L. and Boekelheide, K. (2011) 'Can case study approaches speed implementation of the NRC report 'toxicity testing in the 21 st century: a vision and a strategy', ALTEX, Vol. 28, No. 3, pp.175-182.

Budinsky, R.A., Schrenk, D., Simon, T., Van den Berg, M., Reichard, J.F., Silkworth, J.B., Aylward, L.L., Brix, A., Gasiewicz, T., Kaminski, N., Perdew, G., Starr, T.B., Walker, N.J. and Rowlands, J.C. (2014) 'Mode of action and dose-response framework analysis for receptor-mediated toxicity: the aryl hydrocarbon receptor as a case study', Crit. Rev. Toxicol., Vol. 44, No. 1, pp.83-119.

Corton, J.C., Cunningham, M.L., Hummer, B.T., Lau, C., Meek, B., Peters, J.M., Popp, J.A., Rhomberg, L., Seed, J. and Klaunig, J.E. (2014) 'Mode of action framework analysis for receptor-mediated toxicity: the peroxisome proliferator-activated receptor alpha (PPARalpha) as a case study', Crit. Rev. Toxicol., Vol. 44, No. 1, pp.1-49.

Council of Canadian Academies (CCA) (2012) Integrating Emerging Technologies into Chemical Safety Assessment, Ottawa (ON): The Expert Panel on the Integrated Testing of Pesticides, Council of Canadian Academies [online] http://www.scienceadvice.ca/en/assessments/ completed/pesticides.aspx (accessed 19 April 2016).

Daston, G., Knight, D.J., Schwarz, M., Gocht, T., Thomas, R.S., Mahony, C. and Whelan, M. (2015) 'SEURAT: safety evaluation ultimately replacing animal testing - recommendations for future research in the field of predictive toxicology', Arch. Toxicol., Vol. 89, No. 1, pp.15-23.

Elcombe, C.R., Peffer, R.C., Wolf, D.C., Bailey, J., Bars, R., Bell, D., Cattley, R.C., Ferguson, S.S., Geter, D., Goetz, A., Goodman, J.I., Hester, S., Jacobs, A., Omiecinski, C.J., Schoeny, R., Xie, W. and Lake, B.G. (2014) 'Mode of action and human relevance analysis for nuclear receptor-mediated liver toxicity: a case study with phenobarbital as a model constitutive androstane receptor (CAR) activator', Crit. Rev. Toxicol., Vol. 44, No. 1, pp.64-82. 
Elmore, S.A., Ryan, A.M., Wood, C.E., Crabbs, T.A. and Sills, R.C. (2014) 'FutureTox II: contemporary concepts in toxicology: "pathways to prediction: in vitro and in silico models for predictive toxicology', Toxicol Pathol., Vol. 42, No. 5, pp.940-942.

Gocht, T., Berggren, E., Ahr, H.J., Cotgreave, I., Cronin, M.T., Daston, G., Hardy, B., Heinzle, E., Hescheler, J. and Knight, D.J. (2015) 'The SEURAT-1 approach towards animal free human safety assessment', ALTEX, Vol. 32, No. 1, pp.9-24.

Hamburg, M.A. (2011) ‘Advancing regulatory science', Science, Vol. 331, No. 6020, p.987.

Interagency Coordinating Committee on the Validation of Alternative Methods - ICCVAM (2006a) Addendum to ICCVAM Evaluation of In Vitro Test Methods for Detecting Potential Endocrine Disruptors: Estrogen Receptor and Androgen Receptor Binding and Transcriptional Activation Assays, NIH Publication No. 03-4503 [online] http://ntp.niehs.nih.gov/iccvam/docs/endo_docs/edaddendfinal.pdf (accessed 19 April 2016).

Interagency Coordinating Committee on the Validation of Alternative Methods - ICCVAM (2006b) In Vitro Test Methods for Identifying Ocular Corrosives and Severe Irritants [online] http://ntp.niehs.nih.gov/pubhealth/evalatm/test-method-evaluations/ocular/in-vitro/index.html (accessed 19 April 2016).

Judson, R., Houck, K., Martin, M., Knudsen, T., Thomas, R.S., Sipes, N., Shah, I., Wambaugh, J. and Crofton, K. (2014) 'In vitro and modelling approaches to risk assessment from the US Environmental Protection Agency ToxCast programme', Basic Clin. Pharmacol. Toxicol., Vol. 115, No. 1, pp.69-76.

Knudsen, T.B., Houck, K.A., Sipes, N.S., Singh, A.V., Judson, R.S., Martin, M.T., Weissman, A., Kleinstreuer, N.C., Mortensen, H.M. and Reif, D.M. (2011) 'Activity profiles of 309 ToxCast ${ }^{\mathrm{TM}}$ chemicals evaluated across 292 biochemical targets', Toxicology, Vol. 282, No. 1, pp.1-15.

Krewski, D., Westphal, M., Andersen, M.E., Paoli, G.M., Chiu, W.A., Al-Zoughool, M., Croteau, M.C., Burgoon, L.D. and Cote, I. (2014) 'A framework for the next generation of risk science', Environ. Health Perspect., Vol. 122, No. 8, pp.796-805.

Organisation for Economic Co-operation and Development (OECD) No 168 (2012a) The Adverse Outcome Pathway for Skin Sensitisation Initiated by Covalent Binding to Proteins Part 1: Scientific Evidence, Series on Testing and Assessment No. 168 [online] http://www.oecd.org/ officialdocuments/publicdisplaydocumentpdf/?cote=env/jm/mono(2012)10/part1\&doclanguag $\mathrm{e}=$ en (accessed 19 April 2016).

Organisation for Economic Co-operation and Development (OECD) No. 168 (2012b) The Adverse Outcome Pathway for Skin Sensitisation Initiated by Covalent Binding to Proteins Part 2: Use of the AOP to Develop Chemical Categories and Integrated Assessment and Testing Approaches, Series on Testing and Assessment No. 168 [online] http://www.oecd.org/ officialdocuments/publicdisplaydocumentpdf/?cote=env/jm/mono(2012)10/part2\&doclanguag $\mathrm{e}=\mathrm{en}$ (accessed 19 April 2016).

Organisation for Economic Co-operation and Development (OECD) No. 203 (2014) New Guidance Document on an Integrated Approach on Testing and Assessment (IATA) For Skin Corrosion and Irritation, Series on Testing and Assessment No. 203 [online] http://www.oecd.org/ officialdocuments/publicdisplaydocumentpdf/?cote $=$ env/jm/mono(2014)19\&doclanguage $=$ en (accessed 19 April 2016).

Reif, D.M., Martin, M.T., Tan, S.W., Houck, K.A., Judson, R.S., Richard, A.M., Knudsen, T.B., Dix, D.J. and Kavlock, R.J. (2010) 'Endocrine profiling and prioritization of environmental chemicals using ToxCast data', Environ. Health Perspect., Vol. 118, No. 12, pp.1714-1720.

Reif, D.M., Sypa, M., Lock, E.F., Wright, F.A., Wilson, A., Cathey, T., Judson, R.R. and Rusyn, I. (2013) 'ToxPi GUI: an interactive visualization tool for transparent integration of data from diverse sources of evidence', Bioinformatics, Vol. 29, No. 3, pp.402-403. 
Rovida, C., Alépée, N., Api, A.M., Basketter, D.A., Bois, F.Y., Caloni, F., Corsini, E., Daneshian, M., Eskes, C., Ezendam, J., Fuchs, H., Hayden, P., Hegele-Hartung, C., Hoffmann, S., Hubesch, B., Jacobs, M.N., Jaworska, J., Kleensang, A., Kleinstreuer, N., Lalko, J., Landsiedel, R., Lebreux, F., Luechtefeld, T., Locatelli, M., Mehling, A., Natsch, A., Pitchford, J.W., Prater, D., Prieto, P., Schepky, A., Schüürmann, G., Smirnova, L., Toole, C., van Vliet, E., Weisensee, D. and Hartung, T. (2015) 'Integrated testing strategies (ITS) for safety assessment', ALTEX, Vol. 32, No. 1, pp.25-40.

Russom, C.L., LaLone, C.A., Villeneuve, D.L. and Ankley, G.T. (2014) 'Development of an adverse outcome pathway for acetylcholinesterase inhibition leading to acute mortality', Environ. Toxicol. Chem., Vol. 33, No. 10, pp.2157-2169.

Seidle, T. and Stephens, M.L. (2009) 'Bringing toxicology into the 21st century: a global call to action', Toxicol In Vitro, Vol. 23, No. 8, pp.1576-1579.

Sipes, N.S., Martin, M.T., Kothiya, P., Reif, D.M., Judson, R.S., Richard, A.M., Houck, K.A., Dix, D.J., Kavlock, R.J. and Knudsen, T.B. (2013) 'Profiling 976 ToxCast chemicals across 331 enzymatic and receptor signaling assays', Chem. Res. Toxicol., Vol. 26, No. 6, pp.878-895.

Thomas, R.S., Philbert, M.A., Auerbach, S.S., Wetmore, B.A., Devito, M.J., Cote, I., Rowlands, J.C., Whelan, M.P., Hays, S.M., Andersen, M.E., Meek, M.E., Reiter, L.W., Lambert, J.C., Clewell III, H.J., Stephens, M.L., Zhao, Q.J., Wesselkamper, S.C., Flowers, L., Carney, E.W., Pastoor, T.P., Petersen, D.D., Yauk, C.L. and Nong, A. (2013) 'Incorporating new technologies into toxicity testing and risk assessment: moving from 21 st century vision to a data-driven framework', Toxicol. Sci., Vol. 136, No. 1, pp.4-18.

Tollefsen, K.E., Scholz, S., Cronin, M.T., Edwards, S.W., de Knecht, J., Crofton, K., Garcia-Reyero, N., Hartung, T., Worth, A. and Patlewicz G. (2014) 'Applying adverse outcome pathways (AOPs) to support integrated approaches to testing and assessment (IATA)', Regulatory Toxicology and Pharmacology, Vol. 70, No. 3, pp.629-640.

Wambaugh, J.F., Setzer, R.W., Pitruzzello, A.M., Liu, J., Reif, D.M., Kleinstreuer, N.C., Wang, N.C., Sipes, N., Martin, M., Das, K., DeWitt, J.C., Strynar, M., Judson, R., Houck, K.A. and Lau, C. (2013) 'Dosimetric anchoring of in vivo and in vitro studies for perfluorooctanoate and perfluorooctanesulfonate', Toxicol. Sci., Vol. 136, No. 2, pp.308-327.

Wetmore, B.A., Wambaugh, J.F., Allen, B., Ferguson, S.S., Sochaski, M.A., Setzer, R.W., Houck, K.A., Strope, C.L., Cantwell, K., Judson, R.S., LeCluyse, E., Clewell, H.J., Thomas, R.S. and Andersen, M.E. (2015) 'Incorporating high-throughput exposure predictions with dosimetry-adjusted in vitro bioactivity to inform chemical toxicity testing', Toxicol. Sci., Vol. 148, No. 1, pp.121-136.

Wetmore, B.A., Wambaugh, J.F., Ferguson, S.S., Li, L., Clewell III, H.J., Judson, R.S., Freeman, K., Bao, W., Sochaski, M.A., Chu, T.M., Black, M.B., Healy, E., Allen, B., Andersen, M.E., Wolfinger, R.D. and Thomas, R.S. (2013) 'Relative impact of incorporating pharmacokinetics on predicting in vivo hazard and mode of action from high-throughput in vitro toxicity assays', Toxicol. Sci., Vol. 132, No. 2, pp.327-346.

\section{Notes}

1 On behalf of The Expert Panel on Integrating Emerging Technologies into Chemical Safety Assessment: the Integrated Testing of Pesticides. The full report, including membership of the expert panel, can be found at http://www.scienceadvice.ca/en/assessments/ completed/pesticides.aspx (accessed 19 April 2016). 